\title{
Interaction between microbubble and elastic microvessel in low frequency ultrasound field using finite element method
}

\author{
SHEN YuanYuan ${ }^{1,2}$, WANG TianFu ${ }^{1,2}$, CHIN ChienTing ${ }^{1,2}$, DIAO XianFen ${ }^{1,2} \&$ \\ CHEN SiPing ${ }^{1,2 *}$
}

${ }^{1}$ Guangdong Key Laboratory for Biomedical Measurements and Ultrasound Imaging, Shenzhen 518060, China;

${ }^{2}$ National-Regional Key Technology Engineering Laboratory for Medical Ultrasound, Shenzhen 518060, China

Received April 19, 2012; accepted July 31, 2012; published online November 27, 2012

\begin{abstract}
The interaction between microbubble and elastic microvessel wall has been hypothesized to be important in the mechanisms of therapeutic ultrasound applications. In this study, a 2D axisymmetric finite element numerical model is established to study the interaction between elastic microvessel wall and oscillating microbubble in low frequency ultrasound field using fluid solid interaction method. The numerical results show that the bubble oscillation induces the vessel wall dilation and depression. The von Mises stress distribution over the microvessel wall is heterogeneous and the maximum value of the midpoint on the inner vessel wall could reach 0.23 MPa and 1.32 MPa under PNP 0.2 MPa and 0.5 MPa, respectively. When the bubble collapses, the circumferential stress decreases rapidly and the transmural pressure increases dramatically. Noticeably, the circumferential stress becomes compressive with the maximum magnitude $1.83 \mathrm{MPa}$ under PNP $0.5 \mathrm{MPa}$, larger than the maximum tension value. It is possible that the rapid compression stress during bubble collapse plays important role in mechanical effect on microvessel wall endothelial lining disruption.
\end{abstract}

microbubble, elastic microvessel, finite element method, ultrasound

Citation: Shen Y Y, Wang T F, Chin C T, et al. Interaction between microbubble and elastic microvessel in low frequency ultrasound field using finite element method. Chin Sci Bull, 2013, 58: 291-298, doi: 10.1007/s11434-012-5546-8

Microbubbles have been recently investigated as promising vehicles or agents in therapeutic ultrasound applications such as targeted drug and gene delivery, blood brain barrier disruption $[1,2]$. In low frequency ultrasound field with low mechanical index, the endothelial lining or tight junctions of blood brain barrier were opened reversibly in the presence of microbubbles. Although the microbubble cavitations near elastic biological tissue layer like vessel wall has been proposed as the most important physical mechanism through experimental studies [3], the exact mechanism underlying is not well understood yet.

The oscillation of microbubbles confined to or near rigid wall in response to acoustic field is different from that in large liquid surroundings by experimental observations [4]. However, biological tissues are not rigid. It was observed

*Corresponding author (email: chensiping@ szu.edu.cn) that microbubble expansion was reduced two- to ten-fold when constrained within small compliable vessels compared with oscillations in large vessels using high speed camera [5]. Recently, Chen et al. [6] observed that vessel wall distension and invagination appeared when microbubble expanding and collapsing in ex vivo rat mesentery capillaries. These findings indicated that the bubble-vessel interaction with relation to mechanical properties of biological tissues played important role in the bioeffects associated with microbubble cavitations.

Compared with complex experimental apparatus, numerical simulations have brought increasing attention to studies on microbubble dynamic behavior when constrained by neighboring objects such as rigid boundary or small vessels. Assuming liquid as inviscid flow, preliminary studies investigated small bubble dynamic behavior in rigid tube or near rigid wall [7]. The results showed that the natural frequency 
of a bubble in rigid tube is reduced compared with that in infinite liquid medium [8]. Recently, the effect of vessel elasticity has been incorporated when studying the bubble dynamics confined to capillaries. Qin and Ferrara [9] investigated the acoustic response of a compliant small blood vessel containing a microbubble using a lumped parameter model. The pressure on the inner vessel wall or the transmural pressure across the wall was calculated. The numerical results predicted that the circumferential stress in the microvessel wall exceeded the vascular strength under the ultrasonic field with peak negative pressure of $0.5 \mathrm{MPa}$ and center frequency of $1 \mathrm{MHz}$. The bubble oscillations in a finite length vessel are characterized by high-frequency and low-frequency modes [10]. However, in these two studies the vessel wall compliance was approximated by a nonlinear boundary condition related to intraluminal pressure and the expansion ratio of the vessel radius. The stress distribution could not be analyzed. Coupled finite element and boundary element code was developed to simulate the acoustic response of microbubble in a deformable vessel, but it has limitation by assuming the fluid inviscid and vessel wall linear elastic [11,12].

In the present study, a finite element model using fluid solid interaction method is established to study the interaction between acoustic response of oscillating microbubble and elastic microvessel. Combining the moving mesh method, the stress distribution over the vessel wall and bubble dynamic motion is simulated simultaneously. Implications of the numerical results are expected to provide insight into the mechanism of therapeutic ultrasound microbubble applications.

\section{Numerical model}

\subsection{Mathematical model}

In the present study, a 2D one quarter axisymmetric model is considered as shown in Figure 1. An initially spherical microbubble with radius $1.5 \mu \mathrm{m}\left(R_{0}\right)$ is located at the midpoint of the axis of a long cylindrical microvessel with radius $R_{v}$. The length and thickness of the microvessel $\left(L_{\mathrm{v}}\right)$ are $100 \mu \mathrm{m}$ and $1 \mu \mathrm{m}$, respectively. The acoustic radiation force effect is not considered in the present study. The blood inside the vessel is considered to be a viscous Newtonian incompressible flow. The fluid motion is governed by the Navier-Stokes equations:

$$
\begin{aligned}
\nabla \cdot \boldsymbol{u} & =0, \\
\rho_{1}\left(\frac{\partial \boldsymbol{u}}{\partial t}+(\boldsymbol{u} \cdot \nabla) \boldsymbol{u}\right) & =\nabla \cdot\left(-p \boldsymbol{I}+\mu_{1}\left(\nabla \boldsymbol{u}+\nabla \boldsymbol{u}^{\mathrm{T}}\right)\right),
\end{aligned}
$$

where $\boldsymbol{u}$ is the velocity, $p$ is the pressure, $\boldsymbol{I}$ is the identity tensor, $\rho_{1}$ is the liquid density, and $\mu_{1}$ is the viscosity.

The gas inside the bubble is assumed to obey a polytropic law without diffusion:

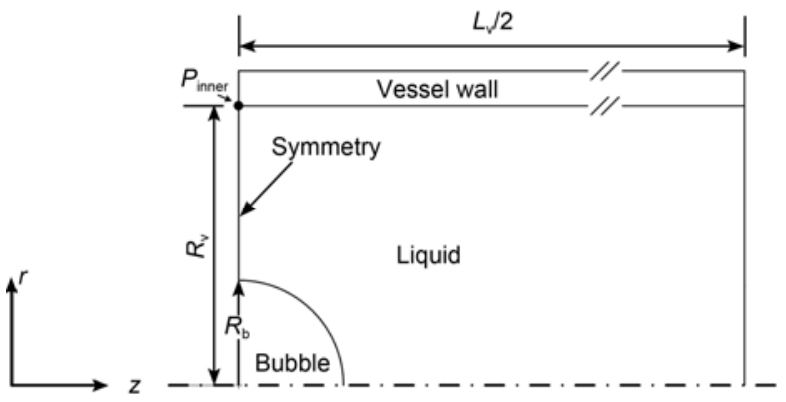

Figure 1 Schematic of geometry model in the simulation.

$$
P_{\mathrm{g}} V^{\gamma}=\text { constant }, \quad V=4 \pi R_{\mathrm{b}}^{3} / 3,
$$

where $P_{\mathrm{g}}$ is the gas pressure inside the microbubble and $V$ is the instantaneous volume of gas, calculated by integration on the gas-liquid moving boundary, $\gamma$ is the polytropic index of gas (1.4) [9]. $R_{\mathrm{b}}$ is defined as the equivalent radius of the bubble for the reason that the bubble dynamic behavior is not spherical. The initial gas pressure inside the microbubble is in balance with the liquid pressure inside the microvessel.

The vessel wall is assumed to be incompressible hyperelastic material using Neo-Hookean model, which is often used to describe biological tissue properties. The strain energy function density $W$ is expressed as

$$
W=\frac{G}{2}\left(\lambda_{1}^{2}+\lambda_{2}^{2}+\lambda_{3}^{2}-3\right) \text {, }
$$

where $G$ is the shear modulus of the vessel wall, and $\lambda_{i}$ is the principal stretch. The parameters and mechanical properties are listed in Table 1 . Since there are no direct measurement data reported about elastic modulus of microvessel wall, it is assumed to be $600 \mathrm{kPa}, 10 \%$ of shear modulus of the artery wall which is composed of organized structure reinforced by elastin and collagen fibers. In order to investigate the effect of different microvessel stiffness, the shear modulus is arbitrarily selected to be $3 \mathrm{MPa}$ or $6 \mathrm{MPa}$, which is 5 -fold or 10 -fold of $600 \mathrm{kPa}$.

Table 1 Parameters and mechanical properties

\begin{tabular}{cll}
\hline Symbol & \multicolumn{1}{c}{ Quantity } & \multicolumn{1}{c}{ Value } \\
\hline$\mu_{\mathrm{l}}$ & liquid viscosity & $0.001 \mathrm{~Pa} \mathrm{~s}$ \\
$\rho_{\mathrm{l}}$ & density of liquid & $1059 \mathrm{~kg} / \mathrm{m}^{3}$ \\
$\rho_{\mathrm{v}}$ & density of vessel wall & $1000 \mathrm{~kg} / \mathrm{m}^{3}$ \\
$G$ & shear modulus of vessel wall & $600 \mathrm{kPa}$ \\
$v$ & Poisson ratio of vessel wall & 0.45 \\
$\sigma$ & surface tension coefficient & $0.07 \mathrm{~N} / \mathrm{m}$ \\
$p_{\mathrm{amb}}$ & ambient pressure & $101325 \mathrm{~Pa}$ \\
$p_{\mathrm{b} 0}$ & the initial blood pressure & $20 \mathrm{mmHg}$ \\
PNP & peak negative pressure of ultrasound & $0.1,0.2 \mathrm{or} 0.5 \mathrm{MPa}$ \\
$f$ & center frequency of ultrasound & $1 \mathrm{MHz}$ \\
\hline
\end{tabular}




\subsection{Boundary conditions}

In this study, the microbubble size is much smaller than the ultrasound wave length. Thus, the wave propagation problem could be neglected. Since the acoustic impedance of the blood is similar with vessel wall, the microvessel end is set as

$$
p_{\text {inlet }}=p_{10}+p_{\text {us }}(t), p_{10}=p_{\text {amb }}+p_{\mathrm{b} 0}
$$

where $p_{10}$ is the initial pressure of the liquid, $p_{\mathrm{amb}}$ is the ambient pressure, $p_{\mathrm{b} 0}$ is the initial blood pressure and $p_{\mathrm{us}}(t)$ is the ultrasound wave pressure. The ultrasound wave is set to be sinusoidal with a center frequency $1 \mathrm{MHz}$, and negative peak arrives first.

Due to the one-quarter model, the symmetry axis is satisfied:

$$
\begin{aligned}
& \boldsymbol{n} \cdot \boldsymbol{u}=0 \\
& \boldsymbol{t} \cdot\left(-p \boldsymbol{I}+\mu_{1}\left(\nabla \boldsymbol{u}+\nabla \boldsymbol{u}^{\mathrm{T}}\right)\right) \boldsymbol{n}=0,
\end{aligned}
$$

where $\boldsymbol{n}$ is the outward-pointing unit normal and $\boldsymbol{t}$ is a tangential vector to the boundary.

On the gas-liquid interface, the normal velocity is continuous and surface tension effect is involved which could not be neglected since microbubble is in micrometer scale:

$$
-\boldsymbol{n} \cdot\left(-p \boldsymbol{I}+\mu_{1}\left(\nabla \boldsymbol{u}+\nabla \boldsymbol{u}^{\mathrm{T}}\right)\right) \cdot \boldsymbol{n}=p_{\mathrm{g}}-\sigma \kappa,
$$

where $\kappa$ is the mean curvature of the gas-liquid interface and $\sigma$ is the surface tension coefficient.

On the vessel wall, the stress and velocity must be continuous due to fluid-structure interaction:

$$
\begin{aligned}
\boldsymbol{u}_{\mathrm{lw}} & =\boldsymbol{u}_{\mathrm{vw}}, \\
\boldsymbol{n} \cdot \boldsymbol{F} & =-p+2 \mu_{1} \frac{\partial u_{i}}{\partial i},
\end{aligned}
$$

where $\boldsymbol{u}_{\mathrm{lw}}$ is the velocity of the liquid wall interface and $\boldsymbol{u}_{\mathrm{vw}}$ is the velocity of vessel wall interface respectively. $\boldsymbol{F}$ is the stress on the vessel wall.

The axial displacements of vessel wall ends are limited to zero. Since microvessels are often embedded in large area of tissue, the outer vessel wall is modeled to be surrounded by liquid to describe the effect.

\subsection{Numerical method}

Numerical simulation is performed using finite element method by COMSOL Multiphysics 3.5a (Sweden AB). Incompressible Navier-Stokes module, axial symmetry stressstrain module and moving mesh module are used to couple the gas-liquid-solid interaction. Triangle elements and quad elements are used to discretize the liquid domain and solid domain respectively, in which the maximum element size is $0.5 \mu \mathrm{m}$ and $0.2 \mu \mathrm{m}$. The maximum mesh element size is
$0.02 \mu \mathrm{m}$ on the gas-liquid interface to get highly precise mean curvature value. The weak form of the boundary equation is used to calculate the mean curvature by applying the divergence theorem on the boundary. The relative tolerance is set to be 0.001 with the time step $0.001 \mu \mathrm{s}$.

\subsection{Validation}

Spherically symmetric bubble motion under acoustically driven field in an infinite liquid environment is well described by Rayleigh-Plesset equation. In order to verify the numerical solution, the spherical microbubble with initial radius $1.5 \mu \mathrm{m}$ is located in the middle of the liquid with a large radius $150 \mu \mathrm{m}$. The shear modulus of the vessel wall is set to be $1 \mathrm{GPa}$. The ultrasonic excitation is one pulse wave with PNP $0.1 \mathrm{MPa}$ and center frequency $1 \mathrm{MHz}$. Then the numerical results are compared with the solution of an unconfined bubble predicted by Rayleigh-Plesset equation solved using Runge-Kutta fourth order method.

Figure 2 shows the expansion ratio of bubble radius variation with time computed by FEM model and RayleighPlesset equation. It shows that the numerical results are in good agreement with the solution of Rayleigh-Plesset equation. The discrepancy between the two results is found to be less than $0.96 \%$.

\section{Results and discussion}

\subsection{The effect of vessel radius and shear modulus on bubble dynamics}

For a microbubble in an elastic microvessel, the vessel radius and elasticity of the wall affect the bubble oscillations. In the present study, the oscillation of microbubble within microvessel with radius $4 \mu \mathrm{m}, 8 \mu \mathrm{m}$ and $16 \mu \mathrm{m}$ are investigated under the ultrasound wave excitation with PNP 0.1 MPa

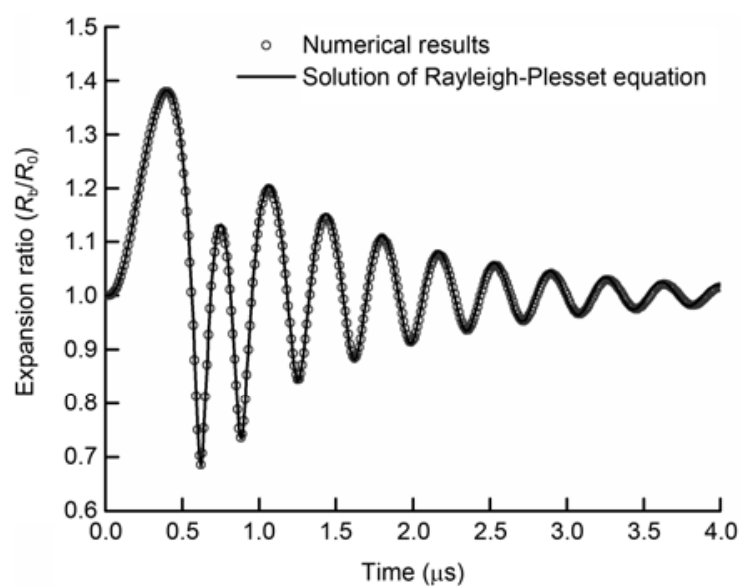

Figure 2 Comparison of the expansion ratio of a microbubble oscillation computed by FEM and predicted by the Rayleigh-Plesset equation. The initial radius of the microbubble is $1.5 \mu \mathrm{m}$. The ultrasonic excitation is one pulse wave with PNP 0.1 MPa and center frequency $1 \mathrm{MHz}$. 
and center frequency $1 \mathrm{MHz}$. Figure 3(a) shows the expansion ratio variations with time, which is defined as the equivalent radius of microbubble $R_{\mathrm{b}}$ normalized by the initial radius $R_{0}$ in the ultrasound field with the shear modulus of vessel wall being $600 \mathrm{kPa}$. As the first negative ultrasound pressure arrives, the microbubble expands from initial radius and reaches its maximum value at time $0.35 \mu \mathrm{s}$, $0.38 \mu$ s and $0.39 \mu$ s with the radius $4 \mu \mathrm{m}, 8 \mu \mathrm{m}$ and $16 \mu \mathrm{m}$, respectively. The maximum expansion ratio does not have significant difference, with the magnitude of 1.34, 1.38 and 1.38 , respectively. The bubble oscillates until the next ultrasound wave pulse comes.

The elasticity of vessel wall is another important parameter influencing microbubble dynamic. In order to evaluate the effect of different elasticity of vessel wall on the microbubble oscillation, the expansion ratios of microbubble in the vessel $\left(R_{\mathrm{v}} 4 \mu \mathrm{m}\right)$ with wall shear modulus $600 \mathrm{kPa}$, $3 \mathrm{MPa}, 6 \mathrm{MPa}$ and rigid vessel wall are compared in Figure 3(b). As shown in this figure, the maximum expansion ratio decreases from 1.39 to 1.23 while the shear modulus increasing from $600 \mathrm{kPa}$ to $6 \mathrm{MPa}$. When the bubble is constrained in rigid vessel, the oscillation appears much slower.

It is demonstrated that the oscillation of microbubbles is constrained within small blood vessels compared with larger surrounding medium by experimental studies [5,13]. Without consideration of fluid viscosity, Miao's numerical results found that the maximum bubble radius, while the radius ratio of vessel to bubble was $16 / 3$, was $\sim 15 \%$ smaller than that for a bubble in an infinite liquid domain when the ultrasound PNP is $0.2 \mathrm{MPa}$ and elastic modulus of vessel wall is $10 \mathrm{MPa}$ [12]. Our results show that the oscillation maximum radius do not have significant differences when the vessel radius is no larger than $16 \mu \mathrm{m}$ under the PNP 0.1 $\mathrm{MPa}$, center frequency $1 \mathrm{MHz}$ and the shear modulus 600 $\mathrm{kPa}$, which is consistent with Qin's results [9]. The results in Figure 3(b) indicate that the expansion degree is also constrained when the vessel wall becomes stiffer. Fung's study indicated that the microvessel elasticity has much relation with the surrounding tissue. It suggests that microbubble dynamic behavior may be different between different organ tissues (e.g. lung, brain), even under the same ultrasound excitation.

Moreover, the oscillation frequency of the microbubble is associated with vessel radius. The oscillation frequency increases while the radius decreases. Martynov et al. investigated the natural frequency of the microbubble with equilibrium radius $4 \mu \mathrm{m}$ in elastic tubes. The results showed that the natural frequency increases by about $35 \%$ when the vessel radius is reduced from 10 to $5 \mu \mathrm{m}$. In our study, with relatively small elasticity $(G<6 \mathrm{MPa})$, the oscillation frequency is affected more by the vessel size rather than the elasticity of the vessel wall, which is similar to the result of Martynov's study. When the vessel wall is rigid, the oscillation frequency is obviously lower than that with elastic vessel wall. However, the oscillation frequency response for bubble in elastic vessel seems different from that in rigid vessel. The natural frequency for bubble in elastic vessels has found to be characterized by multi-frequency mode [10]. Thus the oscillation frequency response for bubble in elastic vessel needs to be further explored in future study.

\subsection{The effect of ultrasound PNP values}

The effect of ultrasound parameters on the motion of the microbubble and microvessel is studied here using multiple PNP values $0.1 \mathrm{MPa}, 0.2 \mathrm{MPa}$ and $0.5 \mathrm{MPa}$. For capillary diameter is usually less than $10 \mu \mathrm{m}$ and ultrasound with center frequency $1-2 \mathrm{MHz}$ is often used in low intensity ultrasound therapeutic applications, the center frequency and microvessel radius is specified to be $1 \mathrm{MHz}$ and $4 \mu \mathrm{m}$, respectively. The shear modulus of the microvessel wall is $600 \mathrm{kPa}$.
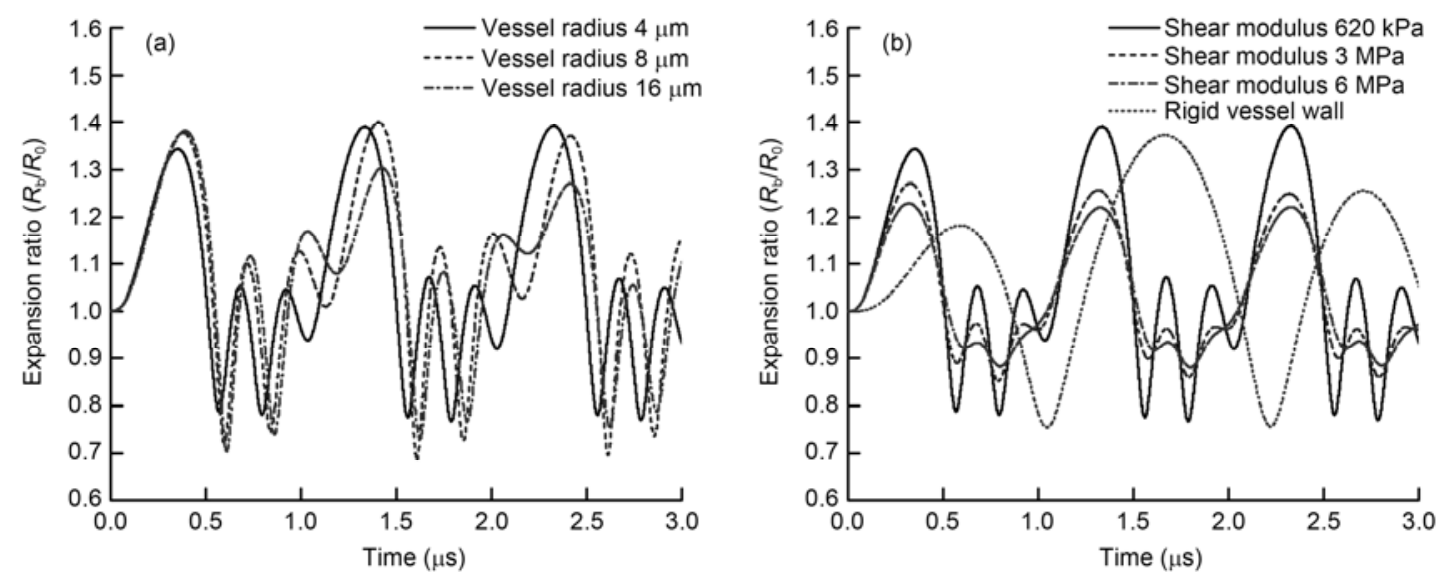

Figure 3 (a) The expansion ratio of the oscillation microbubble within microvessel with radius $4 \mu \mathrm{m}, 8 \mu \mathrm{m}$ and $16 \mu \mathrm{m}$. The shear modulus of vessel wall is $600 \mathrm{kPa}$. (b) The expansion ratio of the oscillation microbubble within vessel $\left(R_{\mathrm{v}} 4 \mu \mathrm{m}\right)$ of shear modulus $600 \mathrm{kPa}, 3 \mathrm{MPa}, 6 \mathrm{MPa}$ and rigid. The ultrasound field is with PNP 0.1 MPa and the center frequency $1 \mathrm{MHz}$. 
Figure 4(a) shows the expansion ratio of the microbubble with PNP 0.1 MPa, 0.2 MPa and 0.5 MPa. The maximum expansion ratio increases from 1.34 to 2.19 and to 4.72 , which is equal to the equivalent radius of the microbubble $2.01 \mu \mathrm{m}, 3.28 \mu \mathrm{m}$ and $7.08 \mu \mathrm{m}$, while PNP increases. The bubble radius decreases quickly due to collapse after increasing to its maximum when PNP 0.2 MPa and 0.5 MPa.

The von Mises stress is commonly used in solid mechanics to predict the yielding of materials. The von Mises stress of the midpoint $P_{\text {inner }}$ on the inner vessel wall (as shown in Figure 1) could be obtained through the numerical simulation using fluid solid interaction method in the present study. Figure 4(b) shows the time history of von Mises stress under PNP 0.1 MPa, 0.2 $\mathrm{MPa}$ and 0.5 MPa. The maximum von Mises stress could reach $0.23 \mathrm{MPa}$ and 1.32 $\mathrm{MPa}$ under PNP 0.2 $\mathrm{MPa}$ and $0.5 \mathrm{MPa}$, which is 5.2-fold and 30-fold of that under PNP 0.1 MPa. After a quick decrease, there appears another quick increase of the stress when the microbubble collapses.

Contour plots of the von Mises stress distribution and the bubble behavior when the equivalent radius reaches its maximum and minimum is depicted in Figure 5. It is clearly seen that the microbubble expansion and collapse induces microvessel dilation and depression. The dynamic behavior of microbubble is nonspherical compared to the case of infinite liquid medium. The maximum stress occurs at the inner vessel wall closest to the microbubble and decreases from the inner wall to the outer wall. The stress distribution types under the three PNP values are similar when the bubble expands. It is shown that the microbubble deflates as shown in Figure 5(d) and (f), indicating jet formation. However, the jet direction is along the axial direction in the case of PNP $0.2 \mathrm{MPa}$, while along the radial direction in the case of PNP 0.5 MPa. In the depression area of the deformed vessel wall, the stress reaches its maximum. However, the minimum stress is located at the transition part from depression to dilation as shown in Figure 5(d) and (f).
Figure 6 shows the contour plots of intraluminal pressure distribution under the ultrasound field with PNP 0.1 MPa, $0.2 \mathrm{MPa}$ and $0.5 \mathrm{MPa}$ at the corresponding times. When the microbubble expands, its volume increases by cubic times and the gas pressure inside decreases dramatically. Such a low-pressure cavitation bubble begins to collapse due to the higher pressure of the surrounding liquid as shown in Figure 6 . Figure 7(a) shows the transmural pressure defined as the difference between the pressure on the midpoint of the inner vessel wall and outer vessel wall. The pressure drop across the vessel wall increases initially as the microbubble expands and then decreases when the equivalent microbubble radius returns to the initial value. However, the transmural pressure increases dramatically during the succeeding collapse. The maximum pressure drop value reaches $0.5 \mathrm{MPa}$ and $2.7 \mathrm{MPa}$ in the case of PNP 0.2 $\mathrm{MPa}$ and $0.5 \mathrm{MPa}$.

During the bubble cavitation, the liquid can generate a positive dynamic pressure on the inner wall surface. The pressure drop across the vessel wall induces the vessel to dilate. The deformation thereby causes stress in the vessel wall. Circumferential stress is usually evaluated to determine the damage mechanisms of vessel rupture. Figure 7(b) shows the circumferential stress of the midpoint on the inner vessel wall. It is seen that the stress increases in the expansion phase while decreases rapidly when collapse occurs under excitation PNP 0.2 MPa, especially 0.5 MPa. A positive value of the stress indicates a tension whereas a negative value indicates a compression. It is noticeable that the circumferential stress becomes compressive during collapsing quickly. The maximum magnitude of the compressive stress reaches $0.55 \mathrm{MPa}$ and $1.83 \mathrm{MPa}$ with PNP 0.2 $\mathrm{MPa}$ and $0.5 \mathrm{MPa}$, even larger than the maximum tension value.

Previous studies focused on comparing the circumferential stress to the tensile strength of the vessel wall to predict the damage threshold. Under ultrasound excitation with PNP 0.2 MPa and center frequency $1 \mathrm{MHz}$, the maximum hoop stress exceeded 1.5 MPa assuming the vessel wall
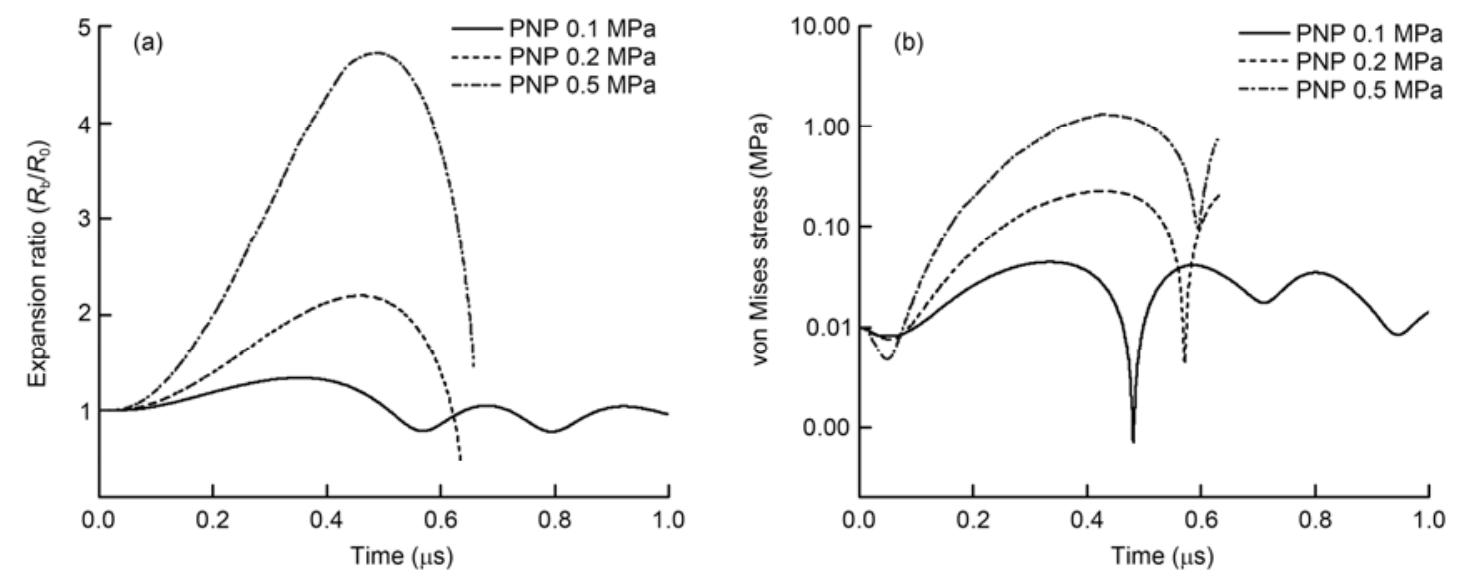

Figure 4 (a) The expansion ratio of the microbubble within vessel under the ultrasound field with PNP 0.1 MPa, $0.2 \mathrm{MPa}$ and $0.5 \mathrm{MPa}$. (b) The von Mises stress of the midpoint on the inner vessel wall under the ultrasound field with PNP $0.1 \mathrm{MPa}$ and $0.2 \mathrm{MPa}$. The initial radius of the microvessel is $4 \mu \mathrm{m}$ and the shear modulus is $600 \mathrm{kPa}$. The center frequency of ultrasound is $1 \mathrm{MHz}$. 

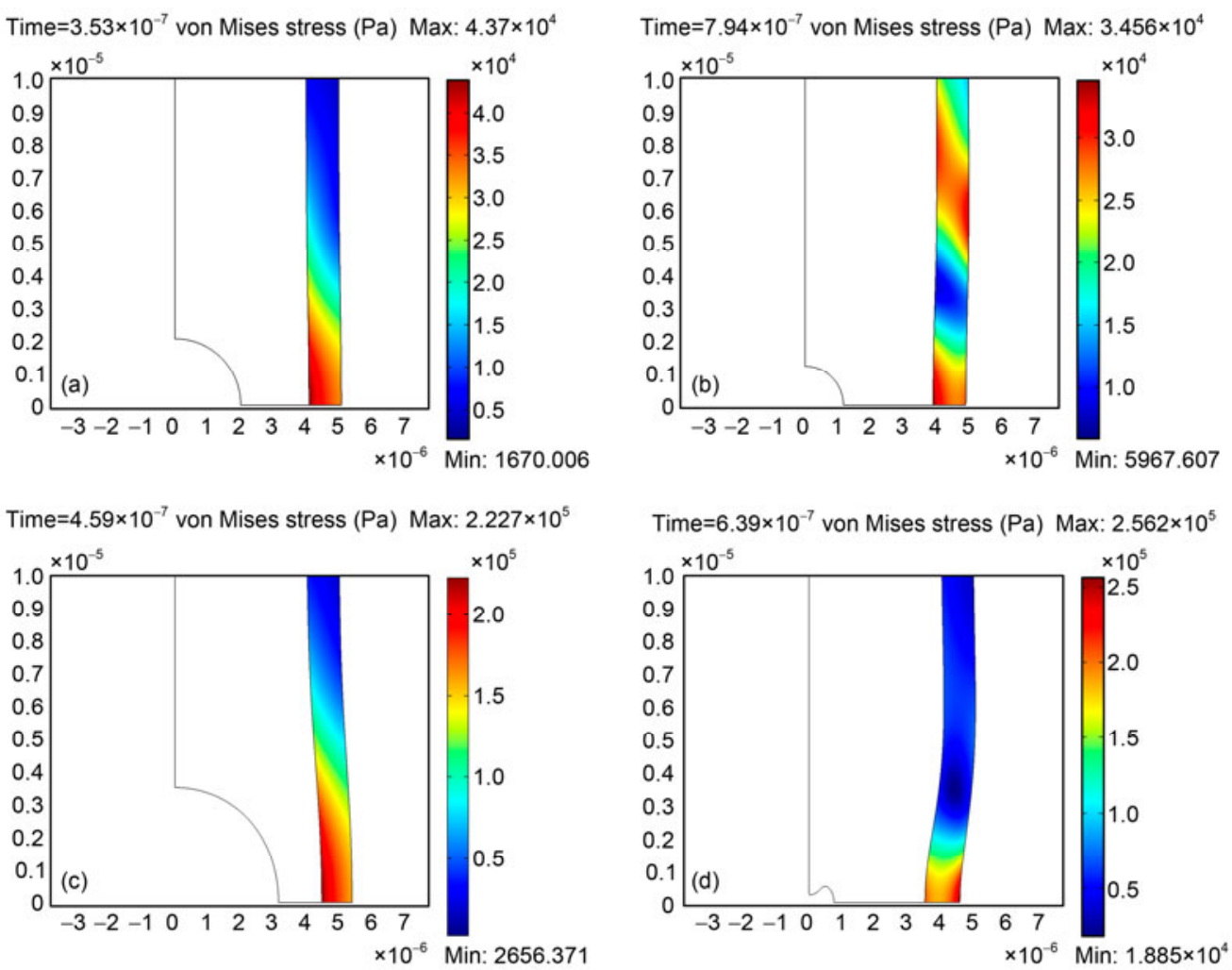

Time $=6.39 \times 10^{-7}$ von Mises stress $(\mathrm{Pa})$ Max: $2.562 \times 10^{5}$
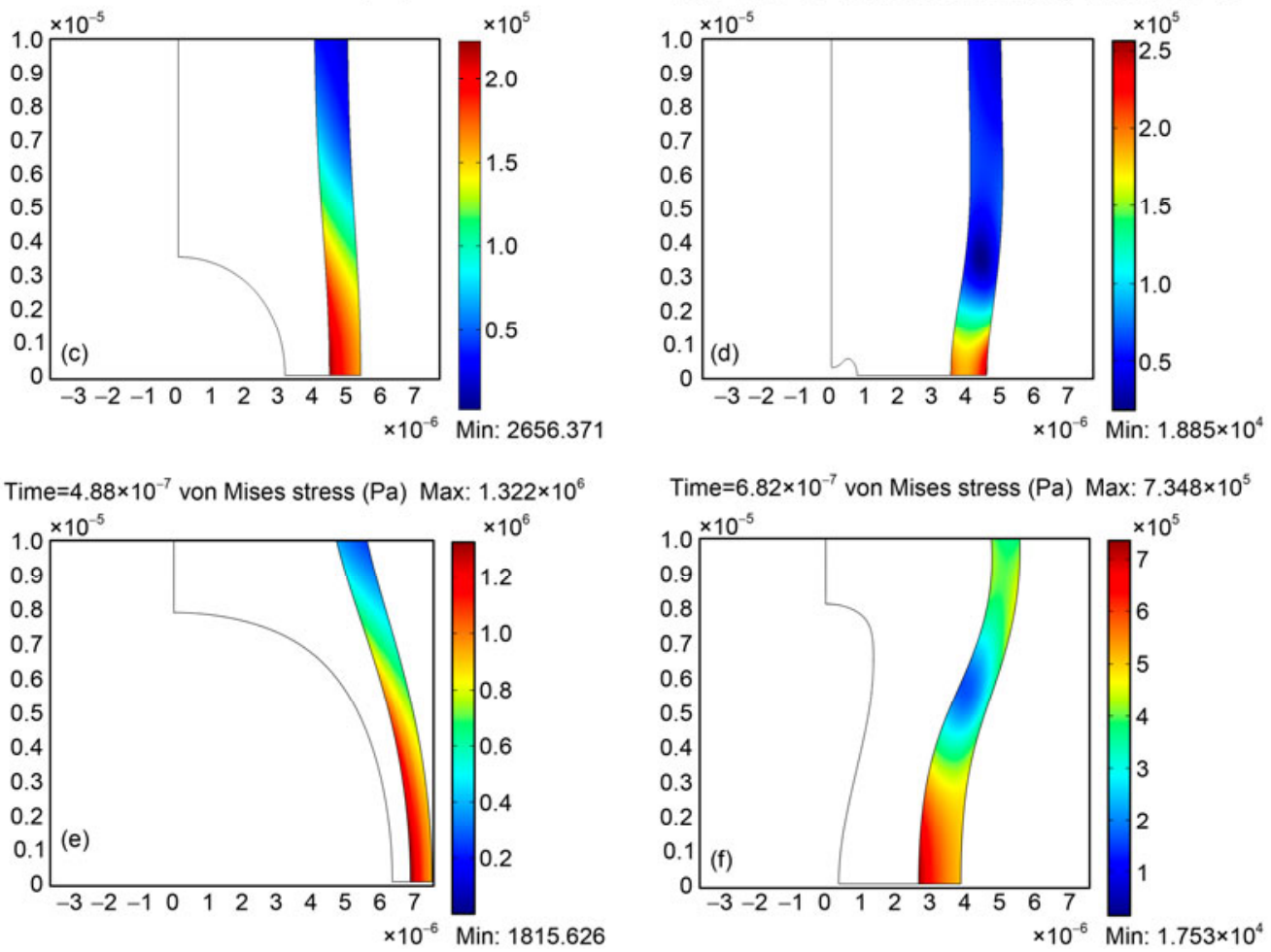

Figure 5 The von Mises stress distribution and the bubble behavior under the ultrasound field PNP $0.1 \mathrm{MPa}((\mathrm{a}),(\mathrm{b})), 0.2 \mathrm{MPa}((\mathrm{c}),(\mathrm{d}))$ and $0.5 \mathrm{MPa}((\mathrm{e})$, (f)) at the time when the equivalent radius is maximum and minimum. The initial radius of the microvessel is $4 \mu \mathrm{m}$ and the shear modulus is $600 \mathrm{kPa}$. The center frequency of ultrasound is $1 \mathrm{MHz}$.

elasticity $5 \mathrm{MPa}[11,12]$. However, it is possible that the rapid compression stress during bubble collapse plays important role in the microvessel wall in ultrasound field. Moreover, the heterogeneous distribution of stress is likely to induce more severe mechanical effects on the vessel wall. Due to fluid solid interaction method, the interaction could be seen clearly. Unlike artery wall, the microvessel structures do not have much elastic or collagen fibers, which could enhance resistance to tensile or compressive strain. There are many studies reported on stress strength of arterial wall but rarely few on microvessel wall, especially on the compressive stress strength.

The mechanical property of the vessel wall was assumed to be linear elastic material in most studies [10-12]. How- ever, Fung's experimental study showed that the stressstrain relationship was nonlinear when a torsion test was made on the mesentery. Unlike large blood vessels being individual tubular structure, microvessels are supported by surrounding tissues [14]. Assuming the microvessel is like a tunnel surrounded by tissue, the shear modulus was measured less than $0.59 \mathrm{MPa}$. In the present study, the vessel wall is assumed to be incompressible hyperelastic material using Neo-Hookean model, which is often used to describe biological tissue properties. However, since microvessels are surrounded by tissues, appropriate model describing mechanical properties of microvessel is needed for more accurate simulation.

Ultrasound microbubbles are encapsulated by shell for 
Time $=3.53 \times 10^{-7}$ Pressure $(\mathrm{Pa})$ Max: $2.422 \times 10^{4}$

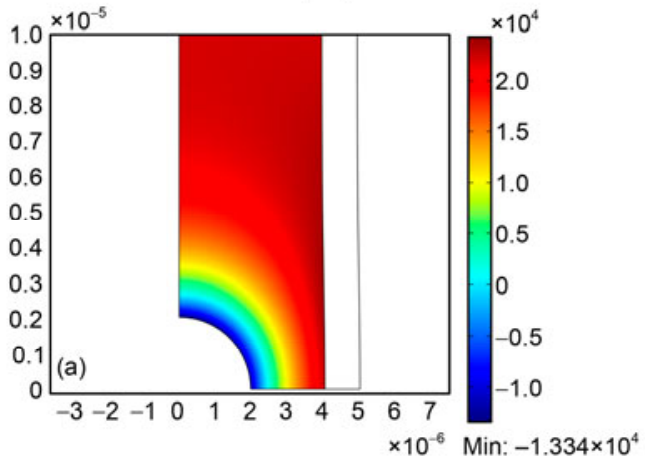

Time $=4.59 \times 10^{-7}$ Pressure $(\mathrm{Pa})$ Max: $6.227 \times 10^{4}$

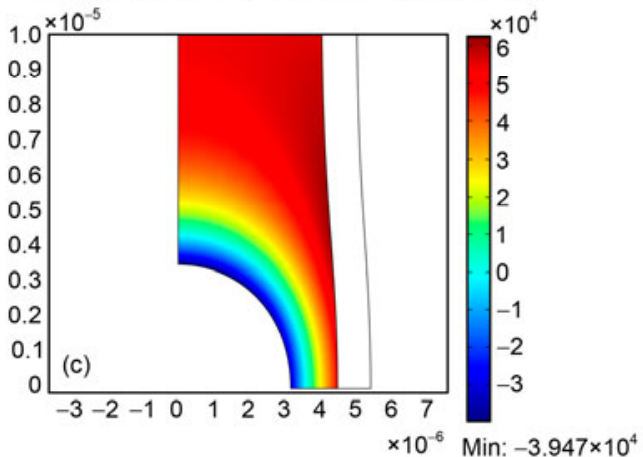

Time $=4.88 \times 10^{-7}$ Pressure $(\mathrm{Pa})$ Max: $1.085 \times 10^{5}$

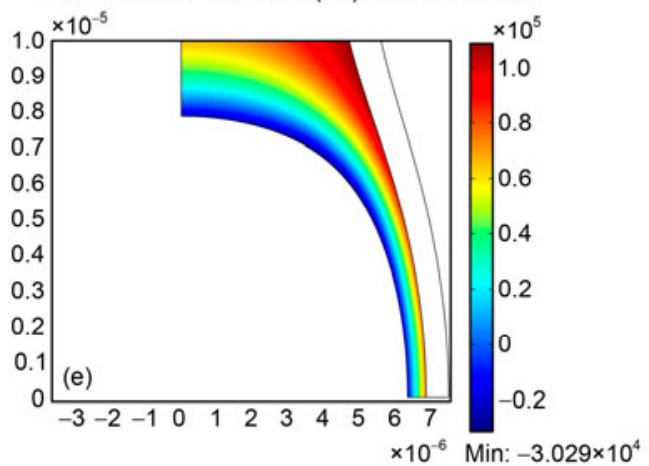

Time $=7.94 \times 10^{-7}$ Pressure (Pa) Max: $4.40 \times 10^{5}$

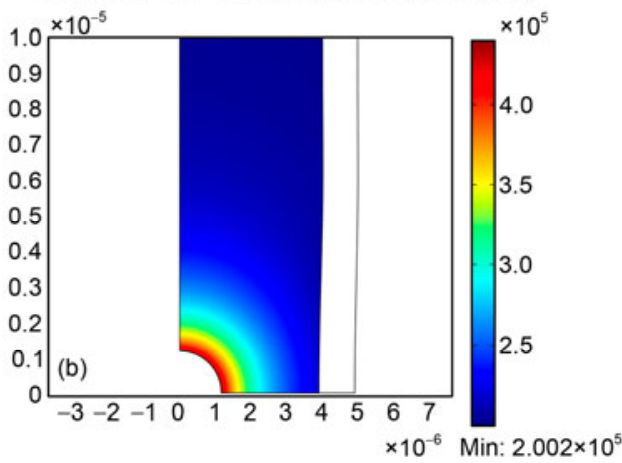

Time $=6.39 \times 10^{-7}$ Pressure $(\mathrm{Pa}) \operatorname{Max}: 7.335 \times 10^{6}$

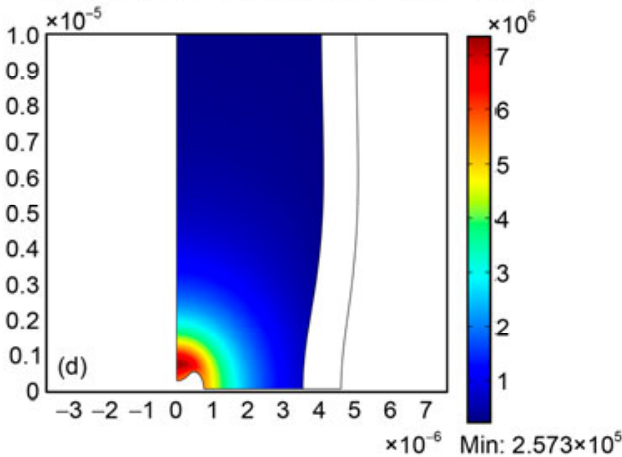

Time $=6.82 \times 10^{-7}$ Pressure $(\mathrm{Pa})$ Max: $7.549 \times 10^{6}$

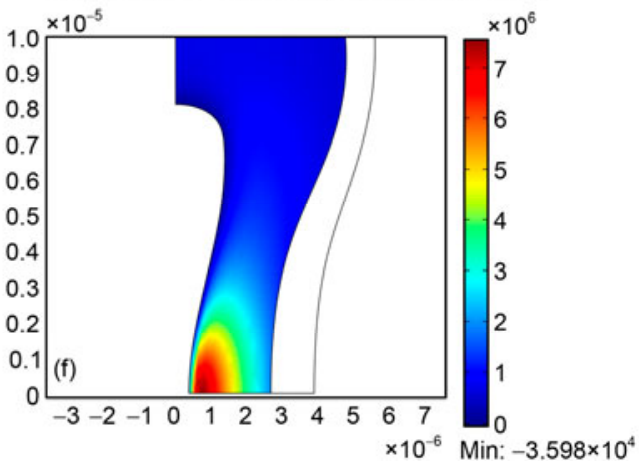

Figure 6 The intraluminal pressure distribution under the ultrasound field with PNP $0.1 \mathrm{MPa}((\mathrm{a}),(\mathrm{b})), 0.2 \mathrm{MPa}((\mathrm{c})$, (d)) and $0.5 \mathrm{MPa}((\mathrm{e})$, (f)) at the time when the equivalent radius is maximum and minimum. The initial radius of the microvessel is $4 \mu \mathrm{m}$ and the shear modulus is $600 \mathrm{kPa}$. The center frequency of ultrasound is $1 \mathrm{MHz}$.
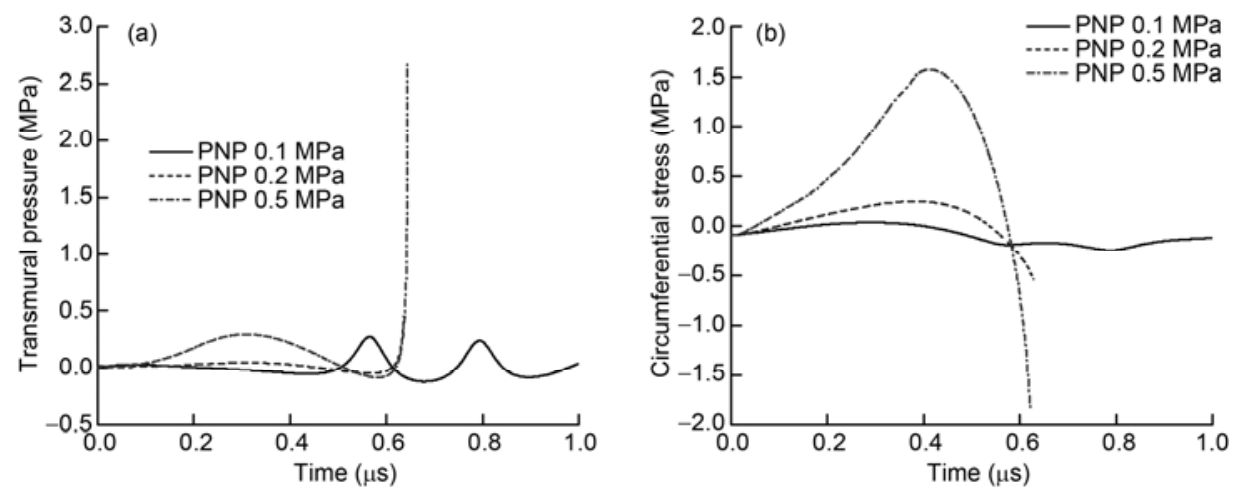

Figure 7 (a) Transmural pressure versus time under the ultrasound field with PNP $0.1 \mathrm{MPa}$ and $0.2 \mathrm{MPa}$. (b) Circumferential stress of the midpoint on the inner vessel wall under the ultrasound field with PNP $0.1 \mathrm{MPa}$ and $0.2 \mathrm{MPa}$. The initial radius of the microvessel is $4 \mu \mathrm{m}$ and the shear modulus is $600 \mathrm{kPa}$. The center frequency of ultrasound is $1 \mathrm{MHz}$. 
stabilization in the biomedical applications nowadays. The shell exerts inertia effect on microbubble oscillation which needs to be further considered. Moreover, since microbubble is in micrometer scale, the surface tension effect is significant. In the present study, the surface tension coefficient is assumed to be constant. However, the surface tension coefficient is varied with bubble radius with lower and upper limits [15], which is needed to be considered in future work.

\section{Conclusion}

A 2D axisymmetric finite element numerical model using fluid solid interaction is established to study the interaction between elastic microvessel wall and oscillating microbubble in low frequency ultrasound field. Numerical results show that the bubble oscillation induces the vessel wall dilation and depression. The von Mises stress distribution over the microvessel wall is heterogeneous and the maximum value of the midpoint on the inner vessel wall could reach $0.23 \mathrm{MPa}$ and 1.32 $\mathrm{MPa}$ under PNP 0.2 $\mathrm{MPa}$ and 0.5 $\mathrm{MPa}$. When the bubble collapses, the circumferential stress decreases rapidly and the transmural pressure increases dramatically. Noticeably, the circumferential stress becomes compressive with the maximum magnitude $1.83 \mathrm{MPa}$ under PNP 0.5 MPa, larger than the maximum tension value. It is possible that the rapid compression stress during bubble collapse plays important role in mechanical effect on microvessel wall endothelial lining disruption.

This work was supported by the National Natural Science Foundation of China (61031003, 81101171), Chinese Postdoctoral Science Foundation (20100470903) and Shenzhen Science and Technology Planning Project (CXB201005240009A).
1 Hynynen K. Macromolecular delivery across the blood-brain barrier. Methods Mol Biol, 2009, 480: 175-185

2 Hynynen K, McDannold N, Vykhodtseva N, et al. Noninvasive MR imaging-guided focal opening of the blood-brain barrier in rabbits. Radiology, 2001, 220: 640-646

3 McDannold N, Vykhodtseva N, Hynynen K. Targeted disruption of the blood-brain barrier with focused ultrasound: Association with cavitation activity. Phys Med Biol, 2006, 51: 793-807

4 Paradossi G, Pellegretti P, Trucco A. Ultrasound Contrast Agents: Targeting and Processing Methods for Theranostics. Milan: Springer, 2011

5 Caskey C F, Stieger S M, Qin S, et al. Direct observations of ultrasound microbubble contrast agent interaction with the microvessel wall. J Acoust Soc Am, 2007, 122: 1191-1200

6 Chen H, Kreider W, Brayman A A, et al. Blood vessel deformations on microsecond time scales by ultrasonic cavitation. Phys Rev Lett, 2011, 106: 034301

7 Sato K, Tomita Y, Shima A. Numerical analysis of a gas bubble near a rigid boundary in an oscillatory pressure field. J Acoust Soc Am, 1994, 95: 2416-2424

8 Oguz H N, Prosperetti A. The natural frequency of oscillation of gas bubbles in tubes. J Acoust Soc Am, 1998, 103: 3301-3308

9 Qin S, Ferrara K W. Acoustic response of compliable microvessels containing ultrasound contrast agents. Phys Med Biol, 2006, 51: 5065-5088

10 Martynov S, Stride E, Saffari N. The natural frequencies of microbubble oscillation in elastic vessels. J Acoust Soc Am, 2009, 126: 2963-2972

11 Miao H, Gracewski S M. Coulpled FEM and BEM code for simulating acoustically excited bubbles near deformable structures. Comput Mech, 2008, 42: 95-106

12 Miao H, Gracewski S M, Dalecki D. Ultrasonic excitation of a bubble inside a deformable tube: implications for ultrasonically induced hemorrhage. J Acoust Soc Am, 2008, 124: 2374-2384

13 Caskey C F, Kruse D E, Dayton P A, et al. Microbubble oscillation in tubes with diameters of 12, 25, and 195 microns. Appl Phys Lett, 2006, 88: 033902

14 Fung Y C, Zweifach B W, Intaglietta M. Elastic environment of the capillary bed. Circ Res, 1966, 19: 441-461

15 Zheng L J, Tu J, Chen W Z. Modeling of ultrasound contrast agents bubble dynamics with modified surface tension coefficient. Chin Sci Bull, 2009, 54: 3501-3507

Open Access This article is distributed under the terms of the Creative Commons Attribution License which permits any use, distribution, and reproduction in any medium, provided the original author(s) and source are credited. 